\title{
Wing-Tip Vortices, Turbulence, and the Distribution of Emissions
}

\author{
Thomas Gerz* and Frank Holzapfel* \\ DLR, German Aerospace Center, D-82234 Wessling, Germany
}

\begin{abstract}
The decay of wing-tip vortices under the influence of turbulence in a stably stratified atmosphere is discussed by means of large-eddy simulations. The vortices originate from a B-747 aircraft in cruise. Atmospheric turbulence and turbulence originating from the boundary layer around the aircraft are distinguished. The former is weak and anisotropic with eddy sizes in the order of the wing span, whereas the latter is wrapped around the vortices with the maximum intensity at the core diameter. During their descent, the parallel vortex tubes approach each other because stratification and turbulence detrain mass into the ambient air. The atmospheric eddies deform the trailing vortices such that their spacing varies. This, in turn, yields different mutually induced velocities that amplify the deformation quickly according to Crow's instability theorem. The bent vortex tubes link after about $1.5 \mathrm{~min}$ and form rings. The continuous trail of turbine exhaust is reorganized in a row of single puffs. Without any atmospheric turbulence the vortices approach each other but remain parallel. They start to dissolve after 2 min when they touch. This dissolution is triggered by the small-scale boundary-layer turbulence. The exhaust trail remains aligned along the flight track.
\end{abstract}

\section{Introduction}

T HE life cycle of the aircraft wake and the exhaust jets is conveniently divided into three phases: During the jet regime, the exhaust from the turbines is entrained into the two counter-rotating wing-tip vortices, which at the same time roll up from the sheet of vorticity around the wings. In the following vortex regime the vortex pair propagates vertically downward by mutual velocity induction. Most of the exhaust is stored in that primary wake and, hence, also sinks below flight level. However, as the primary wake moves downward it produces a wake itself, the so-called secondary wake, because the baroclinic force leads to detrainment of some of the exhaust and leaves a thin curtain of exhaust above the sinking vortex pair. Turbulent mixing increases that detrainment. The vortex regime is followed by the dissipation regime when the organized wing-tip vortices break up into an unorganized (turbulent) wake flow and its energy dissipates to the background level. In that phase the highly concentrated exhaust in the primary wake is suddenly released and diffuses quickly. ${ }^{1}$ Although the principal processes controlling the life of such vortices and the distribution of the turbine exhaust are well known, the effect of turbulence at various scales upon the decay process is rather unclear and joins the role of stable stratification. Both are still subjects of controversy in the literature. ${ }^{2-7}$

Turbulence originates inherently from the unstable vortex sheet behind the trailing edge of the wing, including turbulence from the boundary layer around the airplane and from the exhaust jets. Turbulence also is often a flow state of the surrounding atmosphere. Both kinds, however, differ considerably in strength and length scale. Motivated by the need to assess the impact of aircraft emissions upon the atmospheric state and climate, we consider an ambient flow typical for the height of the tropopause where airliners cruise. There, local zones of wind shear of breaking gravity waves cause turbulence, ${ }^{8,9}$ which is anisotropic and very spotty in space and time. Moreover, the atmosphere is stably stratified at that height most of the time.

In this study we want to investigate how turbulence of both sources (from the aircraft and the atmosphere) affects the decay of the vortex system behind a cruising aircraft in a stably stratified environment. The aircraft under consideration is a B-747. We utilize the method of large-eddy simulations (LES). Similar studies have been undertake ${ }^{10}$ but without discrimination between boundarylayer and ambient turbulence. In a previous paper ${ }^{11}$ the vortex-flow

Received 24 April 1998; presented as Paper 98-2856 at the AIAA 2nd Theoretical Fluid Mechanics Meeting, Albuquerque, NM, 15-18 June 1998; revision received 1 March 1999; accepted for publication 16 April 1999. Copyright (C) 1999 by Thomas Gerz and Frank Holzanpfel. Published by the American Institute of Aeronautics and Astronautics, Inc., with permission.

* Senior Researcher, Institut fur Physik der Atmosphare. behavior and its interaction with the exhaust jets in the near field (jet regime) was simulated and analyzed. Now, we present those LES results that characterize the vortex and the dissipation regimes of wakes exposed to different sources of turbulence and focus mainly on the description of the wake dynamics. In a third paper ${ }^{1}$ we analyzed the diffusion of the exhaust by computing the diffusion and dilution measures throughout the entire wake life cycle.

The LES model and the initial conditions are outlined in Secs. II and III. In Sec. IV we present the main results concerning the role of turbulence on the wake decay and illustrate the distribution of the exhaust in Sec. V. LES data and in situ measurements are compared in Sec. VI. Some conclusions are drawn in Sec. VII.

\section{Numerical Model}

We use a LES code that has been developed for studies of stratified, sheared, and homogeneous turbulence. ${ }^{12}$ The code has been extended to allow for superposition of three-dimensional aircraft wake fields. The LES code works in an Eulerian space-fixed framework and solves the mass conservation equation, the unstationary, incompressible, three-dimensional, Boussinesq-approximated Navier-Stokes equations for the velocity vector $(u, v, w)$, the energy equation for potential temperature $\theta$ as the deviation from the mean background state $\Theta$, and the conservation equation of a dynamically and chemically inactive exhaust concentration, normalized by its maximum initial value $c$. Using the summation convention the equations read, respectively,

$$
\begin{gathered}
\frac{\partial u_{i}}{\partial x_{i}}=0, \quad i=1,2,3 \\
\frac{\partial u_{i}}{\partial t}+\frac{\partial}{\partial x_{j}}\left(u_{j} u_{i}\right)=-\frac{\partial \tau_{i j}}{\partial x_{j}}-\frac{1}{\rho_{0}} \frac{\partial p}{\partial x_{i}}+\beta g \theta \delta_{i 3} \\
\frac{\partial \theta}{\partial t}+\frac{\partial}{\partial x_{j}}\left(u_{j} \theta\right)+\frac{\mathrm{d} \Theta}{\mathrm{d} z} u_{3}=-\frac{\partial \tau_{\theta j}}{\partial x_{j}} \\
\frac{\partial c}{\partial t}+\frac{\partial}{x_{j}}\left(u_{j} c\right)=-\frac{\partial \tau_{c j}}{\partial x_{j}}
\end{gathered}
$$

where $\beta$ and $g$ are the volumetric expansion coefficient and the magnitude of the gravitational acceleration and $\delta_{i j}$ is the Kronecker symbol. The gradient of the mean temperature of the atmospheric background $\mathrm{d} \Theta / \mathrm{d} z$ is constant in space and time.

The subgrid-scale fluxes (denoted by primes) are modeled by Smagorinsky's approach:

$$
\tau_{i j}:=u_{i}^{\prime} u_{j}^{\prime}-\frac{1}{3} u_{i}^{\prime} u_{i}^{\prime} \delta_{i j}=-2 v_{t} S_{i j}
$$


with

$$
\begin{gathered}
S_{i j}=\frac{1}{2}\left(\frac{\partial u_{i}}{\partial x_{j}}+\frac{\partial u_{j}}{\partial x_{i}}\right) \\
\tau_{\theta j}=-\gamma_{t} \frac{\partial \theta}{\partial x_{j}}, \quad \tau_{c j}=-\gamma_{c_{t}} \frac{\partial c}{\partial x_{j}}
\end{gathered}
$$

where $v_{t}=\left(c_{S} \Delta\right)^{2}\left(2 S_{i j} S_{j i}\right)^{1 / 2}$ and $\gamma_{t}=\gamma_{c_{t}}=v_{t} / P r_{\mathrm{SGS}}$ denote the turbulent diffusivities for momentum, heat and species concentration, respectively. The constants $c_{S}=0.165$ and $P r_{\mathrm{SGS}}=0.419$ are set to the theoretical values for isotropic turbulence. When calculating the effective mesh size $\Delta$, a fitting formula ${ }^{13}$ is used to account for the anisotropy of the numerical mesh. Tests of other closures for turbulence decay ${ }^{14}$ have shown that more sophisticated closures are not necessary here because the subgrid-scale energy of the flow (including wake flow and ambient turbulence) amounts initially to less than $5 \%$ of the resolved energy and decays with time. The physical fields are discretized on a staggered grid and integrated in space and time by second-order finite differencing. The integration scheme is nondissipative and only weakly diffusive. All boundaries of the computational domain are periodic.

The domain coordinates are chosen such that the B-747 aircraft with a span of $60 \mathrm{~m}$ flies in the $-x$ direction, the direction $y$ extends from the left to the right side of the aircraft, and $z$ denotes the height. To keep simulation costs at a minimum and the problem size small enough to fit into available computer resources, the size of the computational domain is kept as small as possible and increased in distinct steps when necessary. This need requires a mapping of the fields from a given domain with high resolution into a larger one with coarser resolution. The domain size of the first simulation step covers $L_{x} \times L_{y} \times L_{z}=160 \times 192 \times 180 \mathrm{~m}^{3}$ with a grid volume of $\Delta x \times \Delta y \times \Delta z=2.5 \times 0.5 \times 0.5 \mathrm{~m}^{3}$, respectively. When the wake is $7 \mathrm{~s}$ old, the fields are mapped into a larger domain of size $408 \times 256 \times 540 \mathrm{~m}^{3}$ with a mesh dimension of $6.4 \times 1.0 \times 1.0 \mathrm{~m}^{3}$. A third mapping is processed after $157 \mathrm{~s}$, resulting in a domain of $408 \times 512 \times 640 \mathrm{~m}^{3}$ and a mesh size of $6.4 \times 2.0 \times 2.0 \mathrm{~m}^{3} . L_{x}=408 \mathrm{~m}$ is chosen to cover the most unstable mode for a B-747 according to Crow's theory. ${ }^{15}$ Because the flow varies in flight direction at rather large scales (near-to-homogeneity during jet and vortex regimes), the mesh size $\Delta x$ can be much larger than $\Delta y$ or $\Delta z$. The numerical time step varies between 0.004 and $0.05 \mathrm{~s}$.

Tests showed that the flow was sufficiently resolved in each domain: coarser grids caused unadmissibly high numerical diffusion, whereas a finer grid did not change the vortex properties significantly. Moreover, in our simulations the vortex pair achieves an average sinking speed of about $97.7 \%$ of the theoretical value. The mapping consists of a simple copy of data from every second mesh of the finer grid to every mesh of the coarser grid. Outside the subdomain of the former finer grid, the data are extrapolated in the extended flight direction $x$ but set to zero in $y$ and $z$. The latter causes a layer (one mesh thick) of artificial vorticity inside the new (larger) domain at meshes along the boundaries of the mapped (smaller) domain. This artificial vorticity yielded values around $1 \%$ of the physical vorticity, and, thus, its effect on the flow evolution was neglected. Periodic boundaries led to undesired mirror vortices. The domain, however, is large enough that the vortices are not significantly influenced by their mirror images: The domain size in span direction $L_{y}$ is 4.1 (for $t \leq 7 \mathrm{~s}$ ) and 5.4 (for $t>7 \mathrm{~s}$ ) times larger than the spacing between the two mature vortices. According to other estimates,${ }^{10}$ this size is enough to keep effects of the mirror vortices beyond the periodic boundaries below a few percent.

\section{Initial Conditions}

The simulations cover the period from the early jet regime $(1 \mathrm{~s})$ until the dispersion regime. The exhaust distribution in the early wake, and, therefore, the chemical and microphysical processes involved, is very sensitive to the positions of the turbines relative to the wing tips of an aircraft. ${ }^{16}$ After $1 \mathrm{~s}$ the exhaust plumes are already shifted by several meters below the level of the turbines and toward the evolving vortex centers. At that time the wing-tip vortices are not yet fully rolled up. In contrast to other investigations, we therefore treat vortex roll up and exhaust dilution simultaneously.

The input data are selected such that they resemble a typical case in terms of a cruising aircraft, flight condition, and atmospheric state. ${ }^{17}$ The mean state of our model atmosphere in the tropopause region is motionless but stably stratified with a pressure of $215.9 \mathrm{hPa}$ at $11.3 \mathrm{~km}$ height, a density of $0.35 \mathrm{~kg} / \mathrm{m}^{3}$, and an absolute temperature of $214.3 \mathrm{~K}$ (potential temperature of $332.1 \mathrm{~K}$ ). The temperature gradient of the atmospheric background is in terms of the BruntVaisala frequency $N=(\beta g \mathrm{~d} \Theta / \mathrm{d} z)^{1 / 2}=0.014 \mathrm{~s}^{-1}$.

The B-747 aircraft with a span of $60 \mathrm{~m}$ and a weight of $2.5 \times 10^{6} \mathrm{~N}$ cruises at a speed of $247 \mathrm{~m} / \mathrm{s}$, resulting in a maximum circulation of $\Gamma_{0}=600 \mathrm{~m}^{2} / \mathrm{s}$. The wings have elliptical circulation distributions, i.e., the initial vortex spacing $b_{0}$ is $47 \mathrm{~m}$. Included are also the dynamic, thermodynamic, and gas concentration properties of the exhaust jets from the four turbines. The initial fields of $u, v, w, \theta$, and $c$ have been provided by a vortex filament technique coupled with a jet expansion model. This technique computes the flowfield, which is induced by a series of vortex filaments along the wing (BiotSavart law) and the initial free expansion of the buoyant jets. The technique and the interface with the LES code is described in detail in Ref. 10. When the LES start at a wake age of $1 \mathrm{~s}$, the maximum swirl $V_{C}$ amounts to $12.3 \mathrm{~m} / \mathrm{s}$ at a core radius of $R_{C}=4 \mathrm{~m}$, and the exhaust jet still has a maximum velocity excess of $18 \mathrm{~m} / \mathrm{s}$ being about $10 \mathrm{~K}$ warmer than the ambient air.

In the turbine jets as well as around the aircraft, turbulent boundary layers develop, which quickly are incorporated into the trailing vortices. Like most of the exhaust mass, the largest turbulence fluctuations are situated around the vortex cores at distance $R_{C}$ from the centers. Such turbulence increases mixing and may accelerate the decay process of the wake. In the LES we model the boundary-layer turbulence by adding initially a three-dimensional random perturbation field to the swirling flow such that the perturbation reaches its maximum rms value of $0.16 V_{C}(=2 \mathrm{~m} / \mathrm{s})$ at $R_{C}$ and decays exponentially for smaller and larger radii. This approach became justified recently by Devenport et al., ${ }^{18}$ who measured a laminar flow inside and a turbulent flow just outside the cores of young wing-tip vortices. Likewise, we add a fluctuation to the turbine jets with a maximum of $3 \mathrm{~m} / \mathrm{s}$, and temperature and exhaust concentration fields are not disturbed.

Turbulence in the tropopause region of the atmosphere is very spotty in space and time. Most aircraft encounter turbulence in a rather weak state. The design of atmospheric turbulence in the LES is based upon data reported from the measurement campaign in the North-Atlantic flight corridor near the tropopause. ${ }^{17,19}$ There, in situ measurements show variances of the wind speed of $0.07 \pm 0.03 \mathrm{~m}^{2} \mathrm{~s}^{-2}$ horizontally and $0.011 \pm 0.004 \mathrm{~m}^{2} \mathrm{~s}^{-2}$ vertically when the wind shear ranges between 0.001 and $0.006 \mathrm{~s}^{-1}$. Such a flow state at the atmospheric microscale can be modeled by weak, anisotropic, and decaying turbulence in a stably stratified environment, where almost horizontally oriented eddies cause the mixing. Our initial turbulence fields obey prescribed spectra. To account for the vertical anisotropy of the atmospheric flow at the microscale, we discriminate between horizontal and vertical velocity variance density spectra $E_{i}^{h}$ and $E_{i}^{v}$ for each flow component $i$. The spectra have random phases and read for decaying turbulence ${ }^{20}$ :

$$
\begin{gathered}
E_{j}^{v}\left(k_{3}\right)=C_{j}^{\prime}\left[\frac{1}{2}+2\left(\frac{k_{3}}{k_{p, j}^{v}}\right)^{2}\right] \exp \left[-2\left(\frac{k_{3}}{k_{p, j}^{v}}\right)^{2}\right] \\
E_{3}^{v}\left(k_{3}\right)=C_{3}^{\prime} \exp \left[-2\left(\frac{k_{3}}{k_{p, 3}^{v}}\right)^{2}\right] \\
E_{j}^{h}\left(k_{h}\right)=C_{j}\left[\left(\frac{k_{h}}{k_{p, j}^{h}}\right)^{3}+\frac{1}{2} \frac{k_{h}}{k_{p, j}^{h}}\right] \exp \left[-2\left(\frac{k_{h}}{k_{p, j}^{h}}\right)^{2}\right] \\
E_{3}^{h}\left(k_{h}\right)=C_{3}\left(\frac{k_{h}}{k_{p, 3}^{h}}\right)^{3} \exp \left[-2\left(\frac{k_{h}}{k_{p, 3}^{h}}\right)^{2}\right]
\end{gathered}
$$

where $C_{i}$ contains the mean variances and normalization factors, $k$ and $k_{p}$ are the wave numbers and peak wave numbers, and 
$j=1,2$ denotes the horizontal flow components. The amplitudes $C_{i}$ are set such that the initial (at $t=1 \mathrm{~s}$ ) rms turbulence velocities in the LES are $(\overline{u u})^{1 / 2}=(\overline{v v})^{1 / 2}=0.38 \mathrm{~m} / \mathrm{s}$ horizontally and $(\overline{w w})^{1 / 2}=0.21 \mathrm{~m} / \mathrm{s}$ vertically and, thus, amount to 3.0 and $1.7 \%$ of $V_{C}$. The volume (ensemble)-averaged fluctuating velocities are zero $(\bar{u}=0)$. The chosen peak wave numbers produce horizontal length scales of the most energetic eddies of about $60-90 \mathrm{~m}$, which may represent a typical size when considering the experiences of a slightly bumpy flight. During the initialization step, the atmospheric turbulence fields, which satisfy the continuity condition, are superimposed to the wake fields just described. The initial velocity variances in our LES are larger than the values measured in the North-Atlantic flight corridor in order to account for the rapid decay in the initial transient flow phase in the LES. ${ }^{19}$

To elaborate on the effect of turbulence upon wake decay, three cases are discussed now: no turbulence in case $\mathrm{N}$, aircraft boundarylayer turbulence in case B, and weak atmospheric turbulence (together with boundary-layer turbulence) as the typical case of a bumpy patch along flight tracks at cruising heights in case A. All cases assume a constantly stratified atmosphere. Simulations $\mathrm{N}$ and A terminate at a wake age of $157 \mathrm{~s}$. Case B proceeds until $310 \mathrm{~s}$.

\section{Turbulence and Vortex Decay}

Figure 1 shows the time series of wake and jet flow quantities for cases $\mathrm{N}, \mathrm{B}$, and $\mathrm{A}$ in terms of the maximum values of the velocity components, the pressure minimum as deviation from the ambient pressure, the distance traveled vertically by the vortex pair, and the maximum absolute temperature excess of the exhaust. As long as the vortex cores are intact, these quantities can be attributed to the
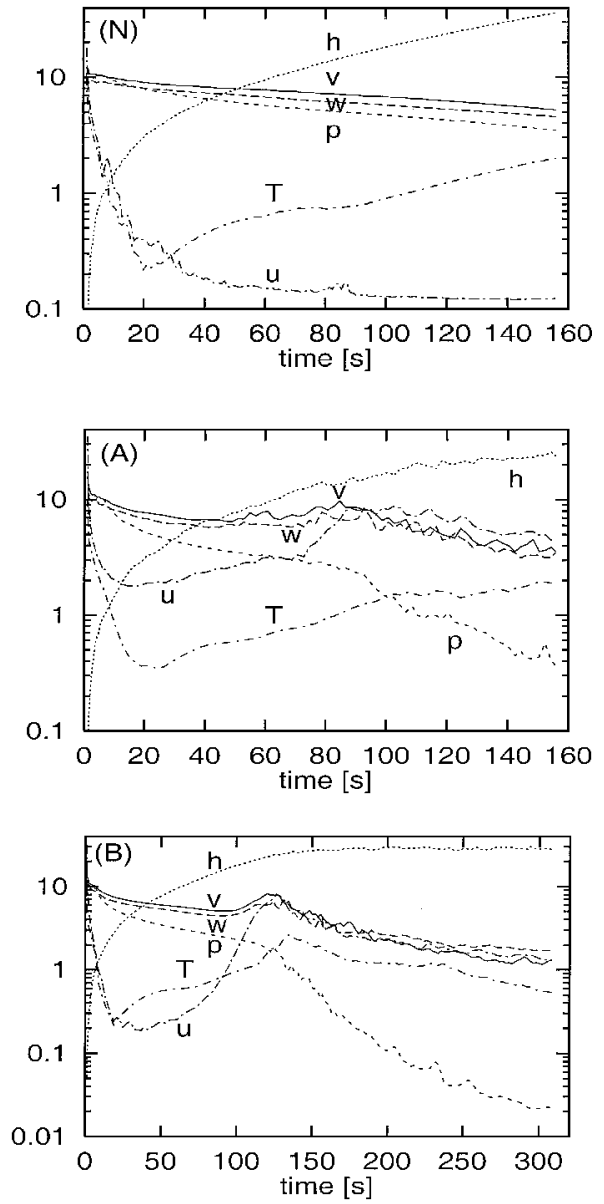

Fig. 1 Time series of wake and jet flow quantities in a stably stratified atmosphere: $\mathrm{N}$, no turbulence; $\mathrm{B}$, boundary-layer turbulence; $\mathrm{A}$, boundary-layer and atmospheric turbulence. Velocity maxima $u, v, w$ $[\mathrm{m} / \mathrm{s}]$; pressure deviation minimum $p(<0)[10 \mathrm{~Pa}]$; downward traveled distance $h[10 \mathrm{~m}]$; maximum of absolute temperature deviation from the background gradient $T[K]$. Note the differently scaled labels for case $\mathbf{B}$. vortex core and the jet dynamics, i.e., $v$ and $w$ define $V_{C}(t)$ at $R_{C}, p$ $(<0)$ marks the center of one of the vortex cores, from its position the traveled distance $h$ is deduced, in the vortex cores the warmest exhaust is found, and $u$ describes its maximum speed. (The component $w$ is smaller than $v$ because, as the upward velocity component, it is reduced by mutual induction of the downward trailing vortex pair.)

In all cases the exhaust jet properties decay very fast initially, whereas the maximum swirl diminishes gradually. The maximal temperature is minimum after $20 \mathrm{~s}$. Here, the jet regime ends because the exhaust is fully trapped now by the wing-tip vortices. After $20 \mathrm{~s}$ the temperature rises again because the vortex pair descends and its mass is heated adiabatically. For thorough discussions of the early wake dynamics and the stratification effects upon the wake vortices, we refer to Refs. 10 and 12, respectively. Here we concentrate on the effect of turbulence.

As the time series reveal, turbulence drastically shortens the lifetime of the organized vortex pair: In case $\mathrm{N}$ the trailing vortex pair reaches the largest descent after $160 \mathrm{~s}(360 \mathrm{~m})$ and still travels downward, whereas in the turbulent cases the vortices level off at 270 and $240 \mathrm{~m}$, respectively. Moreover, in case $\mathrm{N}$ the flow becomes practically two-dimensional because the jet velocity $u$ approaches zero monotonously. Hence, whereas stratification effects can be studied by two-dimensional simulations, the flow is of course three-dimensional when turbulence is present: In cases B and A the turbulent fluctuations trigger a three-dimensional development, visible by the increase of $u$, which amplifies the vortex decay. When $u$ increases, it can no longer be associated with the jet velocity but reflects axial perturbations of the swirl flow, reaching the same magnitude as $v$ and $w$. The onset of the vortex disintegration is also documented by the suddenly stronger increase of the pressure in the cores. In comparison with case B, we see that in the presence of atmospheric turbulence (case A) the vortices start to collapse earlier (after $80 \mathrm{~s}$ instead of $120 \mathrm{~s}$ ).

Figures 2 and 3 depict the wakes of cases B and A in spanheight cross sections. After $87 \mathrm{~s}$ the primary wakes of all cases have traveled downward by $149 \mathrm{~m}$. The descent velocity at that time is $2.2 \mathrm{~m} / \mathrm{s}$. Maxima of temperature (and species concentration) are found around the vortex cores. The relatively warm and buoyant material in the secondary wake moves upward with about $1 \mathrm{~m} / \mathrm{s}$. The atmospheric turbulence event has ceased completely in the meanwhile but was sufficient to trigger strong, largely extended and highly sheared axial fluctuations $u^{4}$. On the other hand, wake B shows only a very weak axial flow, mostly $<0.5 \mathrm{~m} / \mathrm{s}$ at that time (see also Fig. 1). Differences between the states of wakes B and A become very pronounced after $146 \mathrm{~s}$. In case B two counter-rotating vortices with a narrow area of downwash (owing to the reduced vortex spacing) can still be identified, but the organized vortex structure

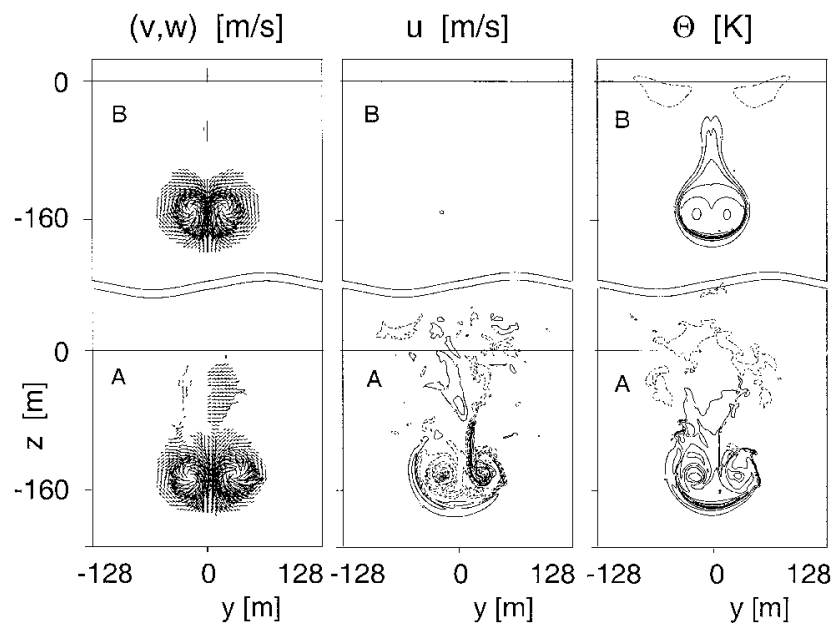

Fig. 2 Instantaneous wakes of case B (top) and A (bottom) after $87 \mathrm{~s}$ in span-height cross sections in terms of swirl velocity $(v, w)$ with magnitude $\geq 1 \mathrm{~m} / \mathrm{s}$; axial velocity $u$ with increment $0.5 \mathrm{~m} / \mathrm{s}$; and temperature deviation $\theta$ with increment $0.2 \mathrm{~K}$ : - - , Negative values, and $\longrightarrow$, the flight level. 

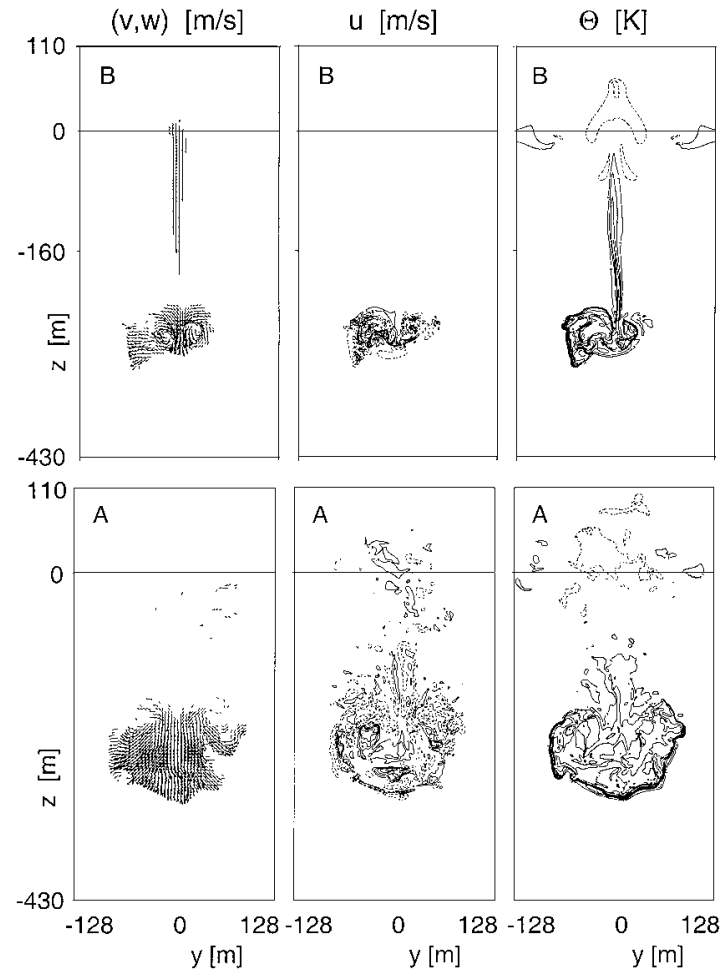

Fig. 3 Wakes of case B (top) and A (bottom) after $146 \mathrm{~s}$. Legend as in Fig. 2.
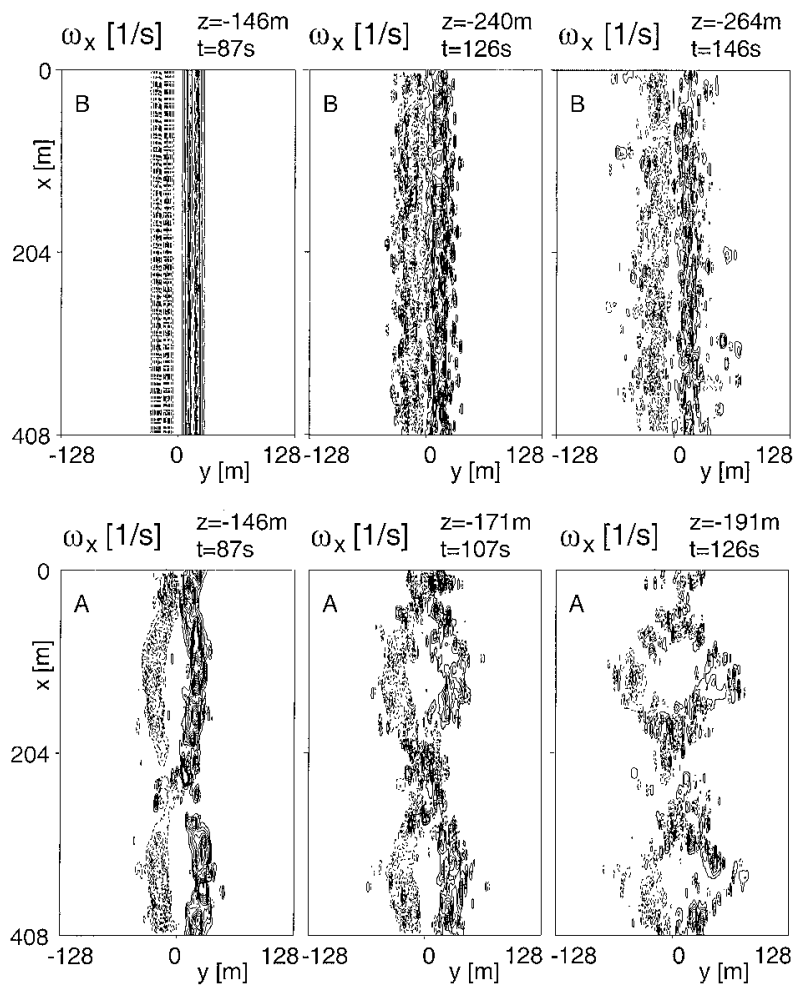

Fig. 4 Span-axial cross sections of axial vorticity $\omega_{x}$ for wakes B (top) and $A$ (bottom) for several instants of time and vertical positions. Contour interval is $0.25 \mathrm{~s}^{-1}$. The left vortex (- - c contours) rotates clockwise.

of case A at that position has dissolved, leaving a large area of a strong downwash. In both cases the downwash flow amounts up to $-4.5 \mathrm{~m} / \mathrm{s}$.

The vorticity component $\omega_{x}$ of cases B and A is shown at various times in Figs. 4 (top views), 5, and 6 (side views). At $87 \mathrm{~s}$ the two vortex tubes in B are still strictly antiparallel. They approach each other more and more, see Fig. 7, and eventually small-scale disturbances appear. On the other hand, the vortices of wake A are already bent and start to link at $87 \mathrm{~s}$. Later, vortex rings form, which still propagate downward. Obviously, we observe the onset and growth

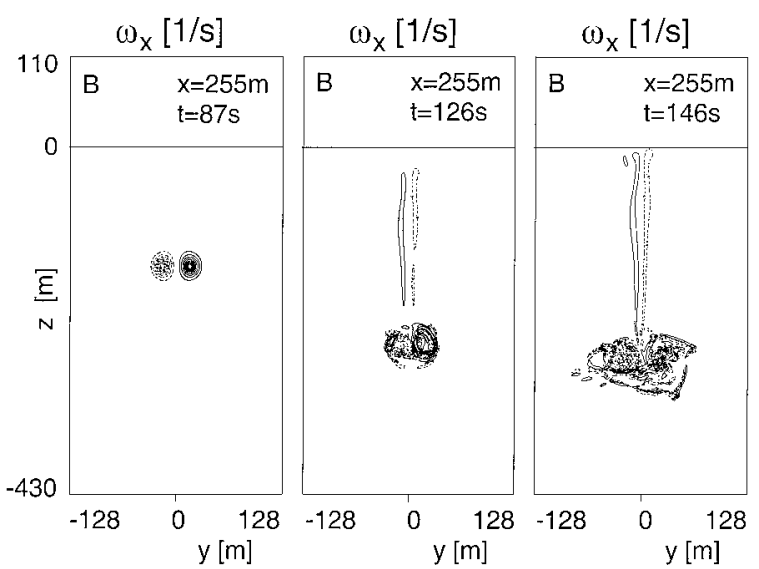

Fig. 5 Span-height cross sections of axial vorticity $\omega_{x}$ for wake $B$ at different times and at axial position $255 \mathrm{~m}$. Contour interval is $0.25 \mathrm{~s}^{-1}$ starting from $\pm 0.125 \mathrm{~s}^{-1}$.
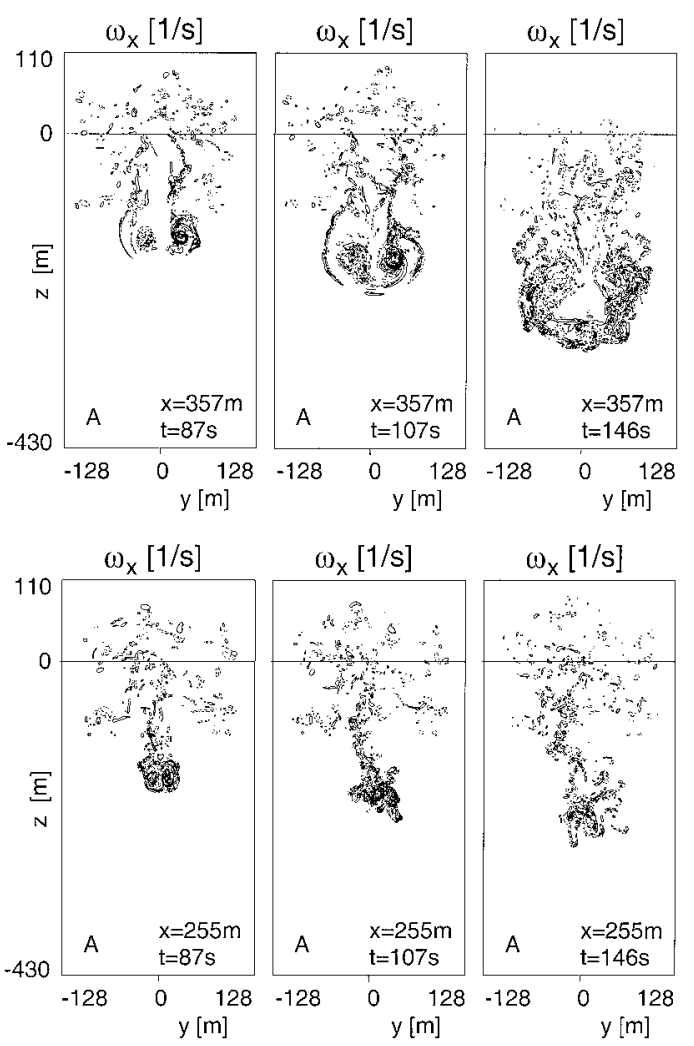

Fig. 6 Span-height cross sections of axial vorticity $\omega_{x}$ for wake $A$ at different times. Vorticity contours are shown at positions where the two vortices have maximum spacing $(x=357 \mathrm{~m}$, upper row $)$ and where they link $(x=255 \mathrm{~m}$, lower row $)$.

of the Crow instability in case A, whereas a larger-scale instability does not evolve in case B. In both cases, however, vorticity of the opposite sign relative to the main vortex is produced outside the primary wake by baroclinity because both wakes propagate downward in a stably stratified atmosphere. ${ }^{5}$ This secondary vorticity travels upward along the primary wake thereby detraining primary mass and exhaust, ${ }^{7}$ which eventually forms the secondary wake up to the flight level.

The primary wake in case B and the surrounding baroclinically produced vorticity manifest a compact and coherent flow ensemble for a longer time than in case A. During that time, the main vortices descend and approach each other. After 2 min, however, wake B suddenly explodes when the core of each primary vortex has grown to half its spacing distance such that the vortices touch; see Fig. 7. Hence, they dissolve by direct interaction of their turbulent cores at small scales. For case A, a similar behavior is observed at $x=255 \mathrm{~m}$ (the linking section), whereas at $x=357 \mathrm{~m}$ (where the distance between the cores is maximum) each arm of the vortex ring seems to dissolve separately. This separate dissolution is triggered when 
Fig. 7 Time series of vortex spacing $b$ (upper plots) and vortex core diameter $D_{c}$.

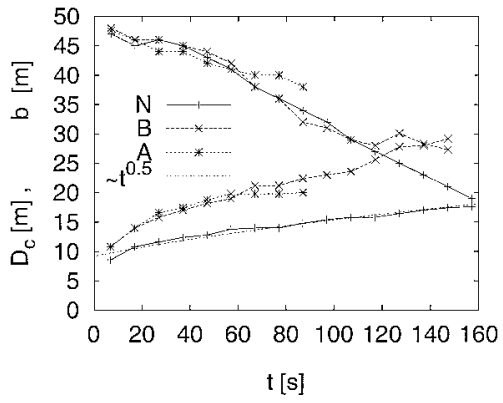

baroclinically produced secondary vorticity is randomly entrained into each main vortex. ${ }^{7}$

Figure 7 displays that in cases N, B, and A the vortices approach each other during their descent while their cores increase. Note that for wake $\mathrm{B}, D_{c}$ and $b$ reach similar values after $2 \mathrm{~min}$, an evidence that the vortices touch. We anticipate a similar touching for case $\mathrm{N}$ after $160 \mathrm{~s}$, however without the subsequent explosion of the vortices because no fluctuations are present to trigger random asymmetries between the two main vortices in that case.

Figure 7 shows further that the core diameter in case $\mathrm{N}$ develops $\sim t^{0.5}$ as predicted by theory. The fit of the growth rate of the core diameter to the analytical solution of the decaying potential vortex delivers an effective Reynolds number based upon circulation of 7400. (Note that the molecular Reynolds number in a LES is infinity.) We remark that the use of numerical advection and diffusion schemes of higher order than second order (which we used) will diminish numerical diffusion, especially inside the vortex cores, and, thus, achieve higher effective Reynolds numbers. Nevertheless, from a very recent comparsion of computer codes (to be published elsewhere) we know that the numerical data presented here and the conclusions drawn are only minimally affected by the Reynolds number.

\section{Exhaust Species Distribution}

The vortex structures in Fig. 4 are similar to the structures of the exhaust plume in Fig. 8, which shows the species concentrations at the respective heights of the primary wake. The exhaust cloud of wake B remains well confined and smooth for 2 min followed by a sudden enlargement when the vortex cores touch and explode. Through the whole evolution the exhaust remains linearly oriented. On the other hand, the evolving large-scale instability in case A interrupts the former line-oriented exhaust and forms almost circular patches in the horizontal projection. Such mammatuslike cloud structures, which are often observed in condensation trails, ${ }^{1,10}$ are always combined with strong downward winds (downdrafts); see the downwash of case A in Fig. 3 that occurs inside a vortex ring (Fig. 4).

\section{Comparison with Measured Data}

The setup of the LES was oriented on in situ measurements in wakes behind B-747 airliners. On 13 November 1994 the DLR research aircraft Falcon encountered the primary wake of a cruising B-747 airplane - clearly noticeable by the accelerations experienced by the Falcon-at the plume age of $82 \mathrm{~s}$ and $145 \mathrm{~m}$ below flight level. ${ }^{21}$ This is in excellent agreement with all simulated cases, which yield $140 \pm 4 \mathrm{~m}$ at that time (Fig. 1). Figure 9 shows that the highest concentrations have been observed and simulated in the primary wake, whereas lower values were sampled in the secondary plume. Moreover, the Falcon measured a temperature excess of $0.8 \mathrm{~K}$ and a $\mathrm{CO}_{2}$ concentration of $4.6 \times 10^{-4} c_{0}$ in the primary wake after $82 \mathrm{~s}$. The simulations yield respective values of $0.7 \mathrm{~K}$ and $2.2 \times 10^{-4} c_{0}$. A more detailed analysis of the exhaust dilution is given in Ref. 1 .

Similar data have been found in 1-to-3-min-old aircraft plumes during other measurements. ${ }^{22}$ Figure 10 displays such data taken when the Falcon crossed a 1-min-old wake of an airliner. In the signals of temperature and (even better) of humidity, the two vortex cores that isolate the trapped exhaust from the ambient air can clearly be recognized. Again the temperature is about $1 \mathrm{~K}$ higher than in the ambient air. This fact puzzled the investigators because
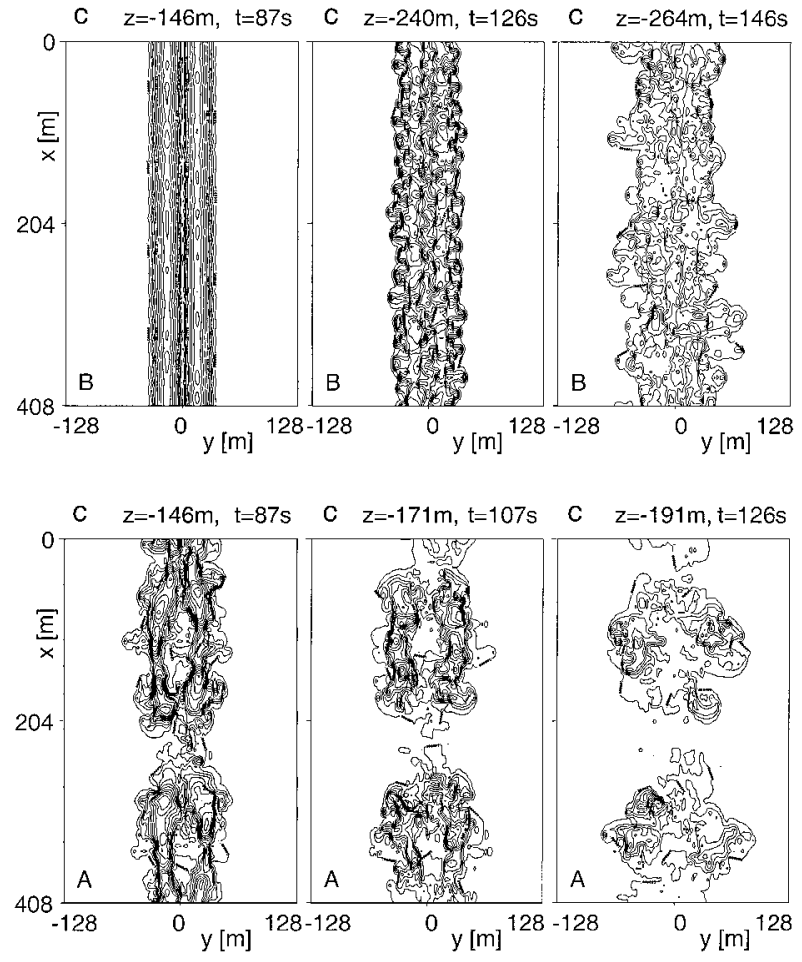

Fig. 8 Span-axial cross sections of species concentrations $c$ of wake B (top) and $A$ (bottom) for several instants of time and vertical positions. Contour increment is $2 \times 10^{-5} c_{0}$ starting at $0.5 \times 10^{-5} c_{0}$.
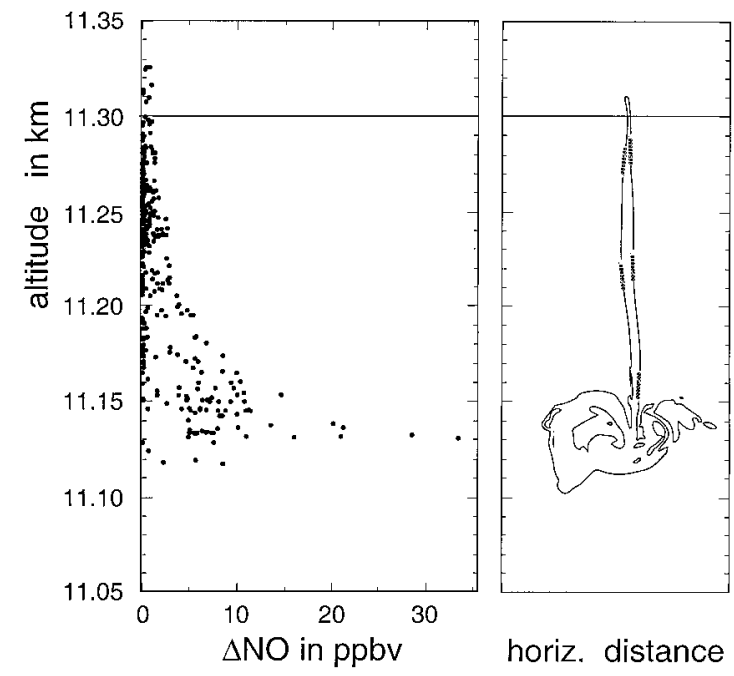

Fig. 9 Exhaust of a B-747 cruising at $11.3 \mathrm{~km}$ height (horizontal line): Left, NO-concentration excesses measured in the wake, ${ }^{21}$ right, simulated exhaust distribution in a vertical cross section perpendicular to the flight direction.

they assumed complete diffusion of the exhaust heat after that time. However, our simulations corroborate the measurement and indicate that this temperature excess is caused by the adiabatic heating of the encapsulated air mass in the primary vortex wake, which trails downward in a stably stratified atmosphere. From the LES data (Fig. 1), we can now predict that even higher temperatures $(2 \mathrm{~K})$ may be measured after $2.5 \mathrm{~min}$ and about $1 \mathrm{~K}$ again in a 4-min-old plume (case B). With the same data ${ }^{22}$ the investigators demonstrated further the evidence for the intact counter-rotating vortex pair, i.e., they measured within or toward the end of the vortex regime and found swirl velocities of $6.5 \mathrm{~m} / \mathrm{s}$. This value matches our LES data, which lie between 5.5 and $7.5 \mathrm{~m} / \mathrm{s}$ after $60 \mathrm{~s}$, very well. Finally, we note that the measured temperature and humidity signals in Fig. 10 indicate, although somewhat scattered, a separation of the two vortices of about $40 \mathrm{~m}$ after $1 \mathrm{~min}$. This again corroborates our LES result of the vortex spacing (see Fig. 7) and, according to our computations, 


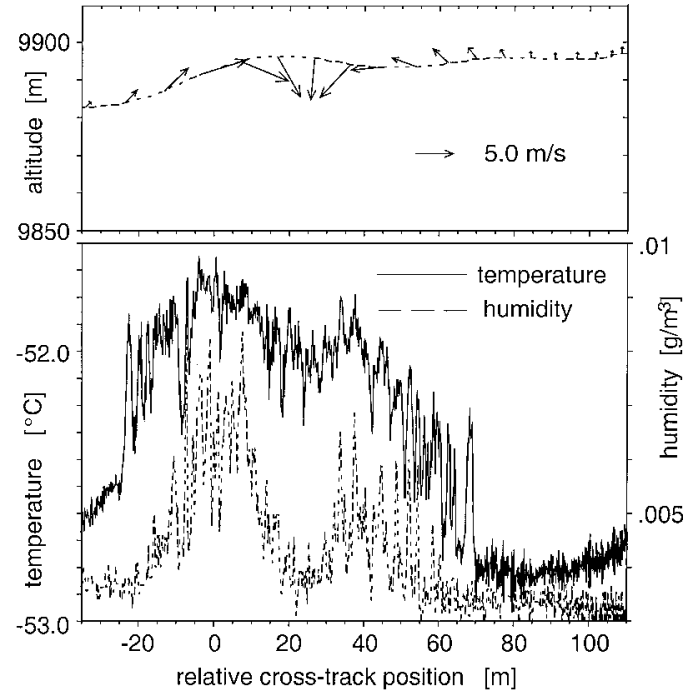

Fig. 10 Properties of a B-747-400 wake at a distance of $13 \mathrm{~km}(\approx 60 \mathrm{~s}){ }^{22}$ Velocity field (upper diagram) and the course of temperature and humidity from a horizontal crossing of the wake.

may indicate that the vortex spacing indeed is reduced during the wake evolution, an issue that is still controversial.

The atmospheric turbulence signature imposed in case A is typical for flight tracks at cruising altitude even in situations with wind shear up to $0.006 \mathrm{~s}^{-1}$ (Refs. 17 and 19); therefore, wake A may resemble a typical case. After $2.5 \mathrm{~min}$ still strong and highly intermittent downwash velocities up to $-4.5 \mathrm{~m} / \mathrm{s}$ prevail inside the vortex rings (see Fig. 3). This may give a hint in explaining pilots' observations of unusually strong turbulence that they encounter when following or crossing the track of an airplane several miles ahead. Finally, the computed turbulence remainder of the vortex flow with peak values of 1-2 m/s in a 5-min-old wake (see case B in Fig. 1) is also corroborated by numerous observations.

Measurements of aircraft wakes in the NASA Ames Research Center wind tunnel revealed a sudden increase in the velocity peaks in the mature wake flow for a short time interval. ${ }^{23}$ Our simulations also show a quick velocity increase in all components during the onset of the vortex collapse (Fig. 1; case B at $120 \mathrm{~s}$, case A at $85 \mathrm{~s}$ ). These peaks originate from the superposition of the mean swirl flow and the growing fluctuations.

\section{Conclusions}

By means of LES, we presented the wake dynamics of a subsonic aircraft (B-747) cruising in a stably stratified atmosphere from wake ages between $1 \mathrm{~s}$ and $5 \mathrm{~min}$. We considered three cases: no turbulence at all $(\mathrm{N})$, aircraft boundary-layer turbulence (B), and weak atmospheric turbulence (A) as a typical case of a slightly bumpy segment along flight tracks in the region of the atmosphere's tropopause. Within that dynamical frame, the distribution of the exhaust concentration has been described qualitatively. The LES show significant differences in the life paths of aircraft wakes depending on the character of imposed turbulence. During the vortex regime, all wakes descend and develop a secondary wake caused by detrainment of air and exhaust mass from the primary wake. The detrainment is caused by baroclinity and enhanced by turbulence. However, size and texture of the secondary wakes and, dynamically more important, the evolutions of the primary wakes differ substantially:

After $1.5 \mathrm{~min}$, wake A is about to lose its primary structure because it starts to become sinusoidally unstable (Crow instability). The vortex tubes link and form rings, and the continuous trail of exhaust (e.g., contrails) is reorganized in a row of single puffs. Therefore, this wake is about to enter the dissipation regime as its primary coherent structure dissolves. Wake B, in contrast, is still in the vortex regime at that time and enters the dissipation regime well after 2 min when the approaching vortex cores touch. It does not experience a large-scale instability, and the exhaust cloud extends almost linearly along the flight track.

We draw the following conclusions.
Compared with the imposed boundary-layer turbulence, the atmospheric perturbations are between 5 and 10 times less intense but have larger length scales (on the order of the aircraft span); therefore, we have to associate the quicker decay of wake A relative to wake B to the action of the atmospheric eddies. They are weak in amplitude but have a length scale that deforms the initially parallel vortex tubes a little by relative advection, which leads to different vortex spacings along the flight path. Once distorted at that scale, the vortex-induced velocities amplify the disturbance according to Crow's instability mechanism, and the primary wake structure dissolves. Because a small axial gradient in vortex spacing is sufficient to trigger the Crow instability, the mechanism is robust to any kind of disturbance as long as the disturbance (background atmospheric turbulence) has the proper length scale of the order of the span of the aircraft.

When an airplane encounters strong atmospheric turbulence patches like in clear-air turbulence, the wing-tip vortices erode earlier and quicker by direct interaction of the vortices with the atmospheric eddies, ${ }^{2}$ possibly triggered by vortex bursting. On the other hand, increasing the boundary-layer turbulence to as much as $60 \%$ of the maximum swirl does not significantly accelerate the decay process because this perturbation remains located at the vortex core radius. Hence, even large disturbances at the scale of the vortex core do not induce unstable modes efficiently. These results seem to corroborate previous studies ${ }^{4}$ where large-scale and smallscale (ambient) turbulence also triggered different mechanisms of decay.

Our LES finally showed that the vortices level off at about $270 \pm 30 \mathrm{~m}$, depending on the state of turbulence. As pointed out by a referee, this result may be of interest with respect to the recent rule change allowing reduced vertical separation minima (RVSM) in the North-Atlantic flight corridor. Airplanes flying East and West are now vertically separated by $305 \mathrm{~m}(1000 \mathrm{ft})$. This nominal separation may vary in reality by a few decades of meters, which, hence, could well explain the recently increasing number of reports of wake-encountering airplanes under cruise.

\section{Acknowledgments}

Parts of this work were supported by Bundesministerium fur Bildung, Wissenschaft, Forschung und Technologie within the project Schadstoffe in der Luftfahrt. The comments of the referees are gratefully acknowledged.

\section{References}

${ }^{1}$ Gerz, T., Durbeck, T., and Konopka, P., "Transport and Effective Diffusion of Aircraft Emissions," Journal of Geophysical Research, Vol. 103, No. D20, 1998, pp. 25,905-25,913.

${ }^{2}$ Spalart, P. R., and Wray, A. A., "Initiation of the Crow Instability by Atmospheric Turbulence," The Characterization and Modification of Wakes from Lifting Vehicles in Fluids, CP-584, AGARD, 1996, pp. 18.1-18.8.

${ }^{3}$ Proctor, F. H., Hinton, D. A., Han, J., Schowalter, D. G., and Lin, Y.-L., "Two-Dimensional Wake Vortex Simulations in the Atmosphere: Preliminary Sensitivity Studies,” AIAA Paper 97-0056, Jan. 1997.

${ }^{4}$ Risso, F., Corjon, A., and Stoessel, A., "Direct Numerical Simulations of Wake Vortices in Intense Homogeneou s Turbulence," AIAA Journal, Vol. 35, No. 6, 1997, pp. 1030-1040.

${ }^{5}$ Spalart, P. R., "Airplane Trailing Vortices," Annual Review of Fluid Mechanics, Vol. 30, 1998, pp. 107-138.

${ }^{6}$ Robins, R. E., and Delisi, D. P., "Numerical Simulation of ThreeDimensional Trailing Vortex Evolution in Stratified Fluid," AIAA Journal, Vol. 36, No. 6, 1998, pp. 981-985.

${ }^{7}$ Holzapfel, F., and Gerz, T., "Two-Dimensional Wake Vortex Physics in the Stably Stratified Atmosphere," Aerospace Science and Technology (to be published); also AIAA Paper 98-2857, 1998.

${ }^{8}$ Dörnbrack, A., and Dürbeck, T., "Turbulent Dispersion of Aircraft Exhausts in Regions of Breaking Gravity Waves," Atmospheric Environment, Vol. 32, No. 18, 1998, pp. 3105-3112.

${ }^{9}$ Schmid, H., and Dornbrack, A., "Simulation of Breaking Gravity Waves During the South Foehn of 7-13 January 1996," Contributions to Atmospheric Physics (to be published).

${ }^{10}$ Lewellen, D. C., and Lewellen, W. S., "Large-Eddy Simulations of the Vortex-Pair Breakup in Aircraft Wakes," AIAA Journal, Vol. 34, No. 11, 1996, pp. 2337-2345.

${ }^{11}$ Gerz, T., and Ehret, T., "Wingtip Vortices and Exhaust Jets During the Jet Regime of Aircraft Wakes," Aerospace Science and Technology, Vol. 1, No. 7, 1997, pp. 463-474. 
${ }^{12}$ Kaltenbach, H.-J., Gerz, T., and Schumann, U., "Large-Eddy Simulation of Homogeneou s Turbulence and Diffusion in Stably Stratified Shear Flow," Journal of Fluid Mechanics, Vol. 280, 1994, pp. 1- 40.

${ }^{13}$ Scotti, A., Meneveau, C., and Lilly, D. K., "Generalized Smagorinsky Model for Anisotropic Grids," Physics of Fluids A, Vol. 5, No. 9, 1993, pp. 2306-2308.

${ }^{14}$ Gerz, T., and Palma, J. M. L. M., "Sheared and Stably Stratified Homogeneous Turbulence: Comparison of DNS and LES," Direct and Large-Eddy Simulation I, edited by P. Voke, L. Kleiser, and J.-P. Chollet, Kluwer, Dordrecht, The Netherlands, 1994, pp. 145-156.

${ }^{15}$ Crow, S. C., "Stability Theory for a Pair of Trailing Vortices," AIAA Journal, Vol. 8, No. 12, 1970, pp. 2172-2179.

${ }^{16}$ Garnier, F., Brunet, S., and Jacquin, L., "Modelling Exhaust Plume Mixing in the Near Field of an Aircraft," Annales Geophysicae, Vol. 15, No. 11, 1997, pp. 1468-1477.

${ }^{17}$ Schumann, U., Konopka, P., Baumann, R., Busen, R., Gerz, T., Schlager, H., Schulte, P., and Volkert, H., "Estimates of Diffusion Parameters of Aircraft Exhaust Plumes near the Tropopause from Nitric Oxide and Turbulence Measurements," Journal of Geophysical Research, Vol. 100, No. D20, 1995 , pp. $14,147-14,162$.

${ }^{18}$ Devenport, W. J., Zsoldos, J. S., and Vogel, C. M., "The Structure and Development of a Counter-Rotating Wing-Tip Vortex Pair," Journal of Fluid Mechanics, Vol. 332, 1997, pp. 71-104
${ }^{19}$ Durbeck, T., and Gerz, T., "The Dispersion of Aircraft Exhausts in the Free Atmosphere," Journal of Geophysical Research, Vol. 101, No. D20, 1996, pp. 26,007-26,015.

${ }^{20}$ Durbeck, T., "Grobstruktursimulation von Mischungsprozessen in der Freien Atmosphare," DLR, German Aerospace Research Center, DLRForschungsberich t 97-02, Cologne, Germany, 1997.

${ }^{21}$ Schulte, P., Schlager, H., Ziereis, H., Schumann, U., Baughcum, S. L., and Deidewig, F. "NO $x$ Emission Indices of Subsonic Long-Range Jet Aircraft at Cruise Altitude: In-Situ Measurements and Predictions," Journal of Geophysical Research, Vol. 102, No. D20, 1996, pp. 21,431-21,442.

${ }^{22}$ Busen, R., Baumann, R., Reinhardt, M. E., Fimpel, H., Kiemle, C., and Quante, M., "Measurements of Physical Properties in the Wake of Commercial Aircraft," Impact of Emissions from Aircraft and Spacecraft upon the Atmosphere, edited by U. Schumann and D. Wurzel, DLR, Mitteilung 94-06, Cologne, Germany, 1994, pp. 297-302.

${ }^{23}$ Rossow, V. J., "Measurements in Vortex Wakes Shed by Conventional and Modified Subsonic Aircraft," The Characterization and Modification of Wakes from Lifting Vehicles in Fluids, CP-584, AGARD, 1996, pp. 26.126.10.

P. Givi Associate Editor 